\title{
Numerical Investigation of Fractional-Order Kawahara and Modified Kawahara Equations by a Semianalytical Method
}

\author{
Weaam Alhejaili, ${ }^{1}$ Sharifah E. Alhazmi, ${ }^{2}$ Rashid Nawaz $\left(\mathbb{D},{ }^{3}\right.$ Aatif Ali $\left(\mathbb{D},{ }^{3}\right.$ \\ Joshua Kiddy Kwasi Asamoah $\mathbb{1}^{4}{ }^{4}$ and Laiq Zada ${ }^{3}$ \\ ${ }^{1}$ Department of Mathematical Sciences, College of Science, Princess Nourah bint Abdulrahman University, P.O. Box 84428, \\ Riyadh 11671, Saudi Arabia \\ ${ }^{2}$ Umm Al-Qura University, College of Education for Girls at Al-Qunfudah, Mathematics Department, Mecca, Saudi Arabia \\ ${ }^{3}$ Department of Mathematics, Abdul Wali Khan University Mardan, Khyber Pakhtunkhwa 23200, Pakistan \\ ${ }^{4}$ Department of Mathematics, Kwame Nkrumah University of Science and Technology, Kumasi, Ghana
}

Correspondence should be addressed to Joshua Kiddy Kwasi Asamoah; topeljoshua@gmail.com

Received 22 December 2021; Revised 8 January 2022; Accepted 17 January 2022; Published 3 February 2022

Academic Editor: Anwar Saeed

Copyright (c) 2022 Weaam Alhejaili et al. This is an open access article distributed under the Creative Commons Attribution License, which permits unrestricted use, distribution, and reproduction in any medium, provided the original work is properly cited.

\begin{abstract}
In this work, the optimal homotopy asymptotic method (OHAM) has been used to find approximate solutions to the nonlinear fractional-order Kawahara and modified Kawahara equations. The method convergence is controlled by a flexible function known as the auxiliary function. The values of the unknown arbitrary constants in the auxiliary function are computed using the Caputo derivative fractional-order and the well-known approach of least squares. Fractional-order derivatives are taken in the Caputo sense with numerical values in the closed interval $[0,1]$. The suggested method is directly applied to fractional-order Kawahara and modified Kawahara equations, with no need for small or large parameter assumptions. The numerical results obtained by the proposed method are compared to the new iterative method (NIM). Results reveal that the proposed method converges faster to the exact solution than other methods in the literature.
\end{abstract}

\section{Introduction}

Fractional computation was established as an important subject of mathematics in 1695. Fractional calculus ideas have recently been successfully expanded to numerous sectors, and academics have increasingly realized that fractional calculus may well reflect many nonlocal occurrences in the fields of natural science and architecture. Rheology, liquid flow, dispersion diffusion transport, dynamic cycles in selfcompatible and porous materials, viscoelasticity, and optics are some of the key areas of fractional calculation today. Very few researchers have drawn on the successful use of fractional systems in these fields to examine their mathematical approximation methods, since diagnostic frameworks are usually difficult to obtain. A variety of real-world problems can be modeled using fractional-order differential equations. These equations have many applications in fluid mechanics, electromagnetic theory, electric grids, diffuse transport, groundwater problems, biological sciences, etc. [1-9]. The exact solution for nonlinear problems is very hard to obtain, and an alternative way is to find the approximate solution. Some familiar approximation methods are used in the series of papers [9-21], etc. Similarly, we extend the well-known optimal homotopy asymptotic method (OHAM) to fractional-order Kawahara and modified Kawahara equations.

The proposed approach was presented by Marinca and Herisanu and applied to resolve nonlinear differential equations in the literature series [22-26]. Recently, Sarwar et al. extended the idea of OHAM fractional-order partial differential equations and used them for different problems having fractional-order derivatives [27, 28]. Nawaz et al. applied the suggested approach to the fractional-order Zakharov-Kuznetsov equations [29]. Likewise, Zada et al. 
applied the proposed approach to various fractional PDEs in the series of articles [30,31]. In this article, the application of OHAM is extended to the modified Kawahara and Kawahara equations together with initial conditions:

$$
\begin{aligned}
& \frac{\partial^{\alpha} \Upsilon(\xi, \tau)}{\partial \tau^{\alpha}}+\Upsilon(\xi, \tau) \frac{\partial \Upsilon(\xi, \tau)}{\partial \xi}+\Upsilon(\xi, \tau)-\frac{\partial^{5} \Upsilon(\xi, \tau)}{\partial \xi^{5}}=0,0<\alpha \leq 1 \\
& \Upsilon(\xi, 0)=\frac{105}{169} \operatorname{sec~}^{4}\left(\frac{\xi}{2 \sqrt{13}}\right)
\end{aligned}
$$

$$
\begin{aligned}
\frac{\partial^{\alpha} \Upsilon(\xi, \tau)}{\partial \tau^{\alpha}} & +\Upsilon^{2}(\xi, \tau) \frac{\partial \Upsilon(\xi, \tau)}{\partial \xi}+\rho \frac{\partial^{3} \Upsilon(\xi, \tau)}{\partial \xi^{3}} \\
& +\mu \frac{\partial^{5} \Upsilon(\xi, \tau)}{\partial \xi^{5}}=0,0<\alpha \leq 1, \\
\Upsilon(\xi, 0) & =\frac{3 \rho}{\sqrt{-10 \mu}} \operatorname{sec~}^{2}(k \xi), k=\frac{1}{2} \sqrt{\frac{-\rho}{2 \mu}} .
\end{aligned}
$$

Here, $\rho$ and $\mu$ are constants. Equations (1) and (2) have become the subject of active and wide research topics in recent times [32-34].

\section{Preliminaries}

Definition 1. The Riemann-Liouville fractional integral operator of an order $\alpha \geq 0$ of a function $G \in C_{\mu}, \mu \geq-1$ is presented by

$$
\left\{\begin{array}{l}
I_{a}^{\alpha} G(\xi)=\frac{1}{\Gamma(\alpha)} \int_{a}^{\xi}(\xi-\mu)^{\alpha-1} G(\mu) d \mu, \alpha>0, \xi>0, \\
I_{a}^{0} G(\xi)=G(\xi) .
\end{array}\right.
$$

Definition 2. The fractional derivative $G(\xi)$ according to Caputo is presented by

$$
\left\{\begin{array}{l}
D_{a}^{\alpha} G(\xi)=I_{a}^{m-\alpha} D^{m} G(\xi)=\frac{1}{\Gamma(m-a)} \int_{a}^{\xi}(\xi-\mu)^{m-\alpha-1} G^{m}(\mu) d \mu, \\
m-1<\alpha \leq m, m \in N, \xi>0, G \in C_{-1}^{m} .
\end{array}\right.
$$

Definition 3. If $m-1<\alpha \leq m, m \in N$, and $G \in C_{\mu}^{m}, \mu \geq-1$, then $D_{a}^{\alpha} I_{a}^{\alpha} G(\xi)=G(\xi)$ and $D_{a}^{\alpha} I_{a}^{\alpha} G(\xi)=G(\xi)-\sum_{k=0}^{m-1} G^{(k)}(\xi-$ a) $/ k !, \xi>0$.

The properties of operator $I^{\alpha}$ are found in $[3,11]$. We introduce the subsequent.

For $G \in C_{\mu}^{m}, \alpha, \beta>0, \mu \geq-1$, and $\gamma \geq-1$,

(1) $I_{a}^{\alpha} G(\xi)$ exist for almost every $\xi \in[a, b]$

(2) $I_{a}^{\alpha} I_{a}^{\beta} G(\xi)=I_{a}^{\alpha+\beta} G(\xi)$

(3) $I_{a}^{\alpha} J_{a}^{\beta} G(\xi)=I_{a}^{\beta} J_{a}^{\alpha} G(\xi)$

(4) $I_{a}^{\alpha}(\xi-a)^{\gamma}=(\Gamma(\gamma+1) / \Gamma(\alpha+\gamma+1))(\xi-a)^{\alpha+\gamma}$

\section{OHAM Methodology to Fractional-Order PDEs [27, 28]}

To extend the basic theory of OHAM for fractional-order PDEs, we assume that the subsequent general fractional differential system

$$
\frac{\partial^{\alpha} \Upsilon(\xi, \tau)}{\partial \tau^{\alpha}}=A(\Upsilon(\xi, \tau)+F(\xi, \tau) \alpha>0
$$

with initial condition

$$
\begin{gathered}
D_{0}^{\alpha-\kappa} \Upsilon(\xi, 0)=h_{\kappa}(r),(\kappa=0,1,2, \cdots, n-1), D_{0}^{\alpha-n} \Upsilon(\xi, 0)=0, n=[\alpha], \\
D_{0}^{\kappa} \Upsilon(\xi, 0)=g_{\kappa}(r),(\kappa=0,1,2, \cdots, n-1), D_{0}^{n} \Upsilon(\xi, 0)=0, n=[\alpha] .
\end{gathered}
$$

In the above equation, $\partial^{\alpha} / \partial \tau^{\alpha}$ represents the Caputo fractional derivative operator, $A$ stands for the differential operator, and $\mathcal{Y}(\xi, \tau)$ represents an unknown function. $F(\xi, \tau)$ is a function that serves as an analytical function.

The homotopy using OHAM for equation (5) is $\phi(\xi$, $\tau ; p): \Omega \times[0,1] \longrightarrow R$ which is satisfied:

$$
\begin{aligned}
& (1-p)\left(\frac{\partial^{\alpha} \phi(\xi, \tau)}{\partial \tau^{\alpha}}-F(\xi, \tau)\right)-H(\xi, p) \\
& \cdot\left(\frac{\partial^{\alpha} \phi(\xi, \tau)}{\partial \tau^{\alpha}}-(A(\phi(\xi, \tau))+F(\xi, \tau))=0 .\right.
\end{aligned}
$$

Hence, $p \in[0,1]$ which is an embedding parameter and $H(\xi, \tau)$ shows the auxiliary function such that

$$
H(\xi, \tau) \neq 0 \text { for } p \neq 0 \text { and } H(\xi, 0)=0 \text {. }
$$

Remark 4. The approximate solution $\phi(\xi, \tau)$ approaches to the closed solution when the numerical values of $p$ varies for 0 to 1 in the closed interval $[0,1]$. The convergence of the OHAM purely depends on the auxiliary function.

The auxiliary function $H(\xi, p)$ is set out below:

$H(\xi, p)=p k_{1}\left(\xi, C_{i}\right)+p^{2} k_{2}\left(\xi, C_{i}\right)+p^{3} k_{3}\left(\xi, C_{i}\right)+\cdots+p^{m} k_{m}\left(\xi, C_{i}\right)$.

In equation (9), $C_{i}, i=1,2, \cdots$, convergence control parameters $k_{i}(\xi), i=1,2, \cdots$, is a function of $\xi$.

By extending $\phi\left(\xi, \tau ; p, C_{i}\right)$ in Taylor's series about $p$, one can obtain

$$
\phi\left(\xi, \tau ; C_{i}\right)=\Upsilon_{0}(\xi, \tau)+\sum_{k=1}^{m} \Upsilon_{k}\left(\xi, \tau ; C_{i}\right) p^{k}, i=1,2,3, \cdots
$$


Putting $p=1$, in the above equation, we have

$$
\left.\Upsilon\left(\xi, \tau ; C_{i}\right)=\Upsilon_{0}(\xi, \tau)+\sum_{k=1}^{\infty} \Upsilon_{k}(\xi, \tau) ; C_{i}\right), i=1,2,3, \cdots
$$

By substituting equation (10) in equation (7) and equating the coefficient of the same powers of $p$, we acquire the series of problems:

$$
\begin{aligned}
& p^{0}: \frac{\partial^{\alpha} \Upsilon_{0}(\xi, \tau)}{\partial \tau^{\alpha}}-F=0, \\
& p^{1}: \frac{\partial^{\alpha} \Upsilon_{1}\left(\xi, \tau, C_{1}\right)}{\partial \tau^{\alpha}}-\left(1+C_{1}\right) \frac{\partial^{\alpha} \Upsilon_{0}(\xi, \tau)}{\partial \tau^{\alpha}} \\
&+\left(1+C_{1}\right) F+C_{1} A\left(\Upsilon_{0}(\xi, \tau)\right)=0 \\
& p^{2}: \frac{\partial^{\alpha} \Upsilon_{2}\left(\xi, \tau, C_{1}, C_{2}\right)}{\partial \tau^{\alpha}}-\left(1+C_{1}\right) \frac{\partial^{\alpha} \Upsilon_{1}\left(\xi, \tau, C_{1}\right)}{\partial \tau^{\alpha}}-C_{2} \frac{\partial^{\alpha} \Upsilon_{0}(\xi, \tau)}{\partial \tau^{\alpha}} \\
&+C_{1} A\left(\Upsilon_{1}\left(\xi, \tau, C_{1}\right)\right)+C_{2}\left(F+A\left(\Upsilon_{0}(\xi, \tau)\right)=0 \cdots\right.
\end{aligned}
$$

The above problems contain fractional-order derivatives. So, we apply the inverse of the operator $I^{\alpha}$ on both sides of the above problems:

$$
\begin{aligned}
& \Upsilon_{0}(\xi, \tau)=I^{\alpha}[F], \\
& \Upsilon_{1}\left(\xi, \tau ; C_{1}\right)=I^{\alpha}\left[\left(1+C_{1}\right) \frac{\partial^{\alpha} \Upsilon_{0}(\xi, \tau)}{\partial \tau^{\alpha}}-\left(1+C_{1}\right) F-C_{1} A\left(\Upsilon_{0}(\xi, \tau)\right)\right], \\
& \Upsilon_{2}\left(\xi, \tau ; C_{1}, C_{2}\right)=I^{\alpha}\left[\left(1+C_{1}\right) \frac{\partial^{\alpha} \Upsilon_{1}\left(\xi, \tau ; C_{1}\right)}{\partial \tau^{\alpha}}+C_{2} \frac{\partial^{\alpha} \Upsilon_{0}(\xi, \tau)}{\partial \tau^{\alpha}}\right. \\
& \left.-C_{1} A\left(\Upsilon_{1}\left(\xi, \tau ; C_{1}\right)\right)-C_{2}\left(F+A\left(\Upsilon_{0}(\xi, \tau)\right)\right)\right] \cdots
\end{aligned}
$$

By using these solutions in equation (11), we obtain the approximate solution:

$$
\tilde{\Upsilon}\left(\xi, \tau ; C_{i}\right)=\Upsilon_{0}(\xi, \tau)+\Upsilon_{1}\left(\xi, \tau ; C_{1}\right)+\Upsilon_{2}\left(\xi, \tau ; C_{1}, C_{2}\right)+\cdots
$$

The residual $R\left(\xi, \tau ; C_{i}\right)$ is acquired by using equation (14) into equation (5).

$C_{1}, C_{2}, \cdots$ can be found by using either the Ritz method, the least squared method, the collocation method, or Galerkin's method. The least-square approach is used here. Here, we introduce the functional

$$
\chi\left(C_{i}\right)=\int_{0}^{t} \int_{\Omega} R^{2}\left(\xi, \tau ; C_{i}\right) d \xi d \tau
$$

then calculate the optimal values for auxiliary constants $C_{i}$ by solving the following equation system:

$$
\frac{\partial \chi}{\partial C_{1}}=\frac{\partial \chi}{\partial C_{2}}=\cdots=\frac{\partial \chi}{\partial C_{m}}=0
$$

3.1. Convergence Theorem. If the series (11) converge to
$\Upsilon(\xi, \tau)$, where $\Upsilon_{k}(\xi, \tau) \in L\left(R^{+}\right)$is generated by the zeroorder system and the $K$-order deformation, then $\mathcal{Y}(\xi, \tau)$ is the exact solution of (5).

Proof. The following series

$$
\sum_{k=1}^{\infty} \Upsilon_{i, k}\left(\xi, \tau ; C_{1}, C_{2}, \cdots, C_{k}\right)
$$

converges and is presented by

$$
\psi_{i}(\xi, \tau)=\sum_{k=1}^{\infty} \gamma_{i, k}\left(\xi, \tau ; C_{1}, C_{2}, \cdots, C_{k}\right),
$$

which satisfies the following:

$$
\lim _{k \rightarrow \infty} \Upsilon_{i, k}\left(\xi, \tau ; C_{1}, C_{2}, \cdots, C_{k}\right)=0
$$

Indeed, the subsequent equation is fulfilled:

$$
\begin{aligned}
\Upsilon_{i, 1}\left(\xi, \tau ; C_{1}\right)+ & \sum_{k=2}^{n} \Upsilon_{i, k}\left(\xi, \tau ; \vec{C}_{k}\right)-\sum_{k=2}^{n} \Upsilon_{i, k-1} \\
& \cdot\left(\xi, \tau ; \vec{C}_{k-1}\right)=\Upsilon_{i, 2}\left(\xi, \tau ; \vec{C}_{2}\right) \\
& -\Upsilon_{i, 1}\left(\xi, \tau ; C_{1}\right)+\cdots+\Upsilon_{i, n}\left(\xi, \tau ; \vec{C}_{n}\right) \\
& -\Upsilon_{i, n-1}\left(\xi, \tau ; \vec{C}_{n-1}\right)=\Upsilon_{i, n}\left(\xi, \tau ; \vec{C}_{n}\right)
\end{aligned}
$$

Now, we have

$$
\begin{aligned}
& L_{i, 1}\left(\Upsilon_{i, 1}\left(\xi, \tau ; C_{1}\right)+\sum_{k=2}^{\infty} L_{1}\left(Y_{i, k}\left(\xi, \tau ; \vec{C}_{k}\right)\right)\right. \\
& \quad-\sum_{k=2}^{\infty} L_{i}\left(\Upsilon_{i, k-1}\left(\xi, \tau ; \vec{C}_{k-1}\right)\right) \\
& =L_{i}\left(\Upsilon_{i, 1}\left(\xi, \tau ; C_{1}\right)\right)+\sum_{k=2}^{\infty} L_{i}\left(Y_{i, k}\left(\xi, \tau ; \vec{C}_{k}\right)\right) \\
& \quad-\sum_{k=2}^{\infty} L_{i}\left(\Upsilon_{i, k-1}\left(\xi, \tau ; \vec{C}_{k-1}\right)\right)=0,
\end{aligned}
$$

which satisfies

$$
\begin{aligned}
& L_{i, 1}\left(\Upsilon_{i, 1}\left(\xi, \tau ; C_{1}\right)+L_{i} \sum_{k=2}^{\infty}\left(\Upsilon_{i, k}\left(\xi, \tau ; \vec{C}_{k}\right)\right)\right. \\
& \quad-L_{i} \sum_{k=2}^{\infty}\left(\Upsilon_{i, k-1}\left(\xi, \tau ; \vec{C}_{k-1}\right)\right) \\
& =\sum_{k=2}^{\infty} C_{m}\left[L _ { i } \left(\left(\Upsilon_{i, k-m}\left(\xi, \tau ; \vec{C}_{k-m}\right)\right)\right.\right. \\
& \left.\quad+N_{i, k-m}\left(\Upsilon_{i, k-1}\left(\xi, \tau ; C_{k-1}\right)\right)\right]+g_{i}(\xi, \tau)=0 .
\end{aligned}
$$


TABle 1: Numerical values of $C_{1}, C_{2}$ for time-fractional Kawahara equation for several values of $\alpha$.

\begin{tabular}{lcc}
\hline$\alpha$ & $C_{1}$ & $C_{2}$ \\
\hline 1.0 & -0.9999983031706354 & 0.000002 .417505787703306 \\
2 & -0.9999609966319342 & 0.000030849221963805824 \\
\hline 3 & & \\
\hline
\end{tabular}

TABle 2: Numerical values of $C_{1}$ for time-fractional modified Kawahara equations for several values of $\alpha$.

\begin{tabular}{lc}
\hline$\alpha$ & $C_{1}$ \\
\hline 1.0 & -0.4647234979611254 \\
2 & -0.9999609966319342 \\
\hline
\end{tabular}

TABLE 3: Comparison of second-order OHAM solution with thirdorder NIM solution for time-fractional Kawahara equation for different values of $\alpha$.

\begin{tabular}{ccccc}
\hline$\xi$ & $\tau$ & $\begin{array}{c}\text { OHAM } \\
\alpha=2 / 3\end{array}$ & $\begin{array}{c}\text { OHAM } \\
\alpha=1\end{array}$ & $\begin{array}{c}\text { Exact } \\
\alpha=1\end{array}$ \\
\hline \multirow{4}{*}{-5} & 0.02 & 0.252877 & 0.253985 & 0.253985 \\
& 0.04 & 0.252017 & 0.253625 & 0.253625 \\
& 0.06 & 0.251298 & 0.253265 & 0.253265 \\
& 0.08 & 0.250657 & 0.252905 & 0.252905 \\
& 0.1 & 0.250069 & 0.252546 & 0.252546 \\
& 0.02 & 0.621292 & 0.621301 & 0.621301 \\
0 & 0.04 & 0.621277 & 0.6213 & 0.6213 \\
& 0.06 & 0.621259 & 0.621298 & 0.621298 \\
& 0.08 & 0.621239 & 0.621295 & 0.621295 \\
& 0.1 & 0.621217 & 0.621291 & 0.621291 \\
& 0.02 & 0.255821 & 0.254707 & 0.254707 \\
& 0.04 & 0.256691 & 0.255068 & 0.255068 \\
& 0.06 & 0.257422 & 0.255429 & 0.255429 \\
& 0.08 & 0.258076 & 0.255791 & 0.255791 \\
& 0.1 & 0.258678 & 0.256153 & 0.256153 \\
\hline
\end{tabular}

Now, if $C_{m}, m=1,2,3, \cdots$, is correctly selected, then the equation leading to

$$
L_{i}\left(\mathcal{Y}_{i}(\xi, \tau)+A=0\right.
$$

is the exact solution.

\section{Main Results}

We test our adopted procedure OHAM for finding the approximate solution of the fractional-order Kawahara equation. For most of the computational work, we used MathType and Mathematica 10.

4.1. Numerical Solution of Fractional Kawahara Equation. First, we assume that the time-fractional Kawahara equation is given in [35]:
TABLE 4: Comparison absolute errors of $2^{\text {nd }}$-order OHAM solution with $3^{\text {rd }}$-order NIM solution for time-fractional Kawahara equations for different values of $\alpha$.

\begin{tabular}{ccccc}
\hline$\xi$ & $\tau$ & $\begin{array}{c}\text { Residual } \\
\alpha=0.5\end{array}$ & $\begin{array}{c}\text { NIM [35] } \\
\alpha=1\end{array}$ & $\begin{array}{c}\text { OHAM } \\
\alpha=1\end{array}$ \\
\hline \multirow{4}{*}{0.02} & $-1.31504 \times 10^{-6}$ & $2.27500 \times 10^{-10}$ & $8.04633 \times 10^{-10}$ \\
& 0.04 & $-3.18365 \times 10^{-6}$ & $1.81933 \times 10^{-9}$ & $1.20722 \times 10^{-9}$ \\
-5 & 0.06 & $-5.04947 \times 10^{-6}$ & $6.13795 \times 10^{-9}$ & $8.08547 \times 10^{-10}$ \\
& 0.08 & $-6.91298 \times 10^{-6}$ & $1.45438 \times 10^{-8}$ & $7.88584 \times 10^{-10}$ \\
& 0.1 & $-8.77447 \times 10^{-6}$ & $2.83953 \times 10^{-8}$ & $3.97934 \times 10^{-9}$ \\
& 0.02 & $-8.08613 \times 10^{-8}$ & $1.76636 \times 10^{-13}$ & $1.29552 \times 10^{-12}$ \\
& 0.04 & $-2.14003 \times 10^{-7}$ & $2.82618 \times 10^{-12}$ & $3.06177 \times 10^{-12}$ \\
0 & 0.06 & $-3.84142 \times 10^{-7}$ & $1.43068 \times 10^{-11}$ & $1.05937 \times 10^{-12}$ \\
& 0.08 & $-5.84492 \times 10^{-7}$ & $4.52161 \times 10^{-11}$ & $2.1665 \times 10^{-11}$ \\
& 0.1 & $-8.11039 \times 10^{-7}$ & $1.10391 \times 10^{-10}$ & $7.35924 \times 10^{-11}$ \\
& 0.02 & $1.33389 \times 10^{-6}$ & $2.27670 \times 10^{-10}$ & $8.05429 \times 10^{-10}$ \\
5 & 0.04 & $3.22731 \times 10^{-6}$ & $1.82204 \times 10^{-9}$ & $1.20837 \times 10^{-9}$ \\
& 0.06 & $5.12378 \times 10^{-6}$ & $6.15166 \times 10^{-9}$ & $8.03524 \times 10^{-10}$ \\
& 0.08 & $7.02284 \times 10^{-6}$ & $1.45871 \times 10^{-8}$ & $8.16471 \times 10^{-10}$ \\
& 0.1 & $8.92419 \times 10^{-6}$ & $2.85011 \times 10^{-8}$ & $4.061 \times 10^{-9}$ \\
\hline & & & &
\end{tabular}

$$
\frac{\partial^{\alpha} \Upsilon(\xi, \tau)}{\partial \tau^{\alpha}}+\Upsilon(\xi, \tau) \frac{\partial \Upsilon(\xi, \tau)}{\partial \xi}+\frac{\partial^{3} \Upsilon(\xi, \tau)}{\partial \xi^{3}}-\frac{\partial^{5} \Upsilon(\xi, \tau)}{\partial \xi^{5}}=0,0<\alpha \leq 1
$$

Subject to I.C.,

$$
\Upsilon(\xi, 0)=\frac{105}{169} \operatorname{sech}^{4}\left(\frac{\xi}{2 \sqrt{13}}\right)
$$

For $\alpha=1$, an exact solution for equation (24) is found by [35] as

$$
\Upsilon(\xi, \tau)=\frac{105}{169} \operatorname{sech}^{4}\left(\frac{1}{2 \sqrt{13}}\left(\xi-\frac{36 \tau}{169}\right)\right)
$$

Recall the OHAM preparation given in Section 3, we obtain the subsequent problems:

Zero-order problem:

$$
\frac{\partial^{\alpha} \Upsilon_{0}(\xi, \tau)}{\partial \tau^{\alpha}}=0, \Upsilon_{0}(\xi, \tau)=\frac{105}{169} \operatorname{sech}^{4}\left(\frac{\xi}{2 \sqrt{13}}\right)
$$

First-order problem:

$$
\begin{aligned}
\frac{\partial^{\alpha} \Upsilon_{1}(\xi, \tau)}{\partial \tau^{\alpha}}= & \frac{\partial^{\alpha} \Upsilon_{0}(\xi, \tau)}{\partial \tau^{\alpha}}+C_{1} \frac{\partial^{\alpha} \Upsilon_{0}(\xi, \tau)}{\partial \tau^{\alpha}}-C_{1} \Upsilon_{0} \frac{\partial \Upsilon_{0}(\xi, \tau)}{\partial \xi} \\
& +C_{1} \frac{\partial^{3} \Upsilon_{0}(\xi, \tau)}{\partial \xi^{3}}-C_{1} \frac{\partial^{5} \Upsilon_{0}(\xi, \tau)}{\partial \xi^{5}}
\end{aligned}
$$


TABLE 5: Comparison of $1^{\text {st }}$-order OHAM solution with $3^{\text {rd }}$-order NIM solution for time-fractional modified Kawahara equations For different values of $\alpha$.

\begin{tabular}{|c|c|c|c|c|c|}
\hline$\xi$ & $\tau$ & Exact solution & OHAM solution & $\begin{array}{l}\text { Absolute error NIM for [35] } \\
\qquad \alpha=1\end{array}$ & Absolute error OHAM for $\alpha=1$ \\
\hline \multirow{5}{*}{-5} & 0.02 & $9.474889415 \times 10^{-4}$ & $9.474984314 \times 10^{-4}$ & $9.48992 \times 10^{-9}$ & $9.48992 \times 10^{-9}$ \\
\hline & 0.04 & $9.474794138 \times 10^{-4}$ & $9.474984314 \times 10^{-4}$ & $1.90176 \times 10^{-8}$ & $1.90176 \times 10^{-8}$ \\
\hline & 0.06 & $9.474698483 \times 10^{-4}$ & $9.474984314 \times 10^{-4}$ & $2.8583 \times 10^{-8}$ & $2.8583 \times 10^{-8}$ \\
\hline & 0.08 & $9.474602454 \times 10^{-4}$ & $9.474984314 \times 10^{-4}$ & $3.81862 \times 10^{-8}$ & $3.81862 \times 10^{-8}$ \\
\hline & 0.1 & $9.474506042 \times 10^{-4}$ & $9.474984314 \times 10^{-4}$ & $4.78271 \times 10^{-8}$ & $4.78271 \times 10^{-8}$ \\
\hline \multirow{5}{*}{0} & 0.02 & $9.486832790 \times 10^{-4}$ & $9.486832980 \times 10^{-4}$ & $1.89737 \times 10^{-11}$ & $1.89737 \times 10^{-11}$ \\
\hline & 0.04 & $9.486832221 \times 10^{-4}$ & $9.486832980 \times 10^{-4}$ & $7.58947 \times 10^{-11}$ & $7.58947 \times 10^{-11}$ \\
\hline & 0.06 & $9.486831272 \times 10^{-4}$ & $9.486832980 \times 10^{-4}$ & $1.70763 \times 10^{-10}$ & $1.70763 \times 10^{-10}$ \\
\hline & 0.08 & $9.486829944 \times 10^{-4}$ & $9.486832980 \times 10^{-4}$ & $3.03579 \times 10^{-10}$ & $3.03579 \times 10^{-10}$ \\
\hline & 0.1 & $9.486828237 \times 10^{-4}$ & $9.486832980 \times 10^{-4}$ & $4.74342 \times 10^{-10}$ & $4.74342 \times 10^{-10}$ \\
\hline \multirow{5}{*}{5} & 0.02 & $9.475078835 \times 10^{-4}$ & $9.474984314 \times 10^{-4}$ & $9.45216 \times 10^{-9}$ & $9.45216 \times 10^{-9}$ \\
\hline & 0.04 & $9.475172979 \times 10^{-4}$ & $9.474984314 \times 10^{-4}$ & $1.88666 \times 10^{-8}$ & $1.88666 \times 10^{-8}$ \\
\hline & 0.06 & $9.475266744 \times 10^{-4}$ & $9.474984314 \times 10^{-4}$ & $2.82432 \times 10^{-8}$ & $2.82432 \times 10^{-8}$ \\
\hline & 0.08 & $9.475360132 \times 10^{-4}$ & $9.474984314 \times 10^{-4}$ & $3.75821 \times 10^{-8}$ & $3.75821 \times 10^{-8}$ \\
\hline & 0.1 & $9.475453144 \times 10^{-4}$ & $9.474984314 \times 10^{-4}$ & $4.68832 \times 10^{-8}$ & $4.68832 \times 10^{-8}$ \\
\hline
\end{tabular}

Second-order problem:

$$
\begin{aligned}
& \frac{\partial^{\alpha} \Upsilon_{2}(\xi, \tau)}{\partial \tau^{\alpha}}=\frac{C_{2} \partial^{\alpha} \Upsilon_{0}(\xi, \tau)}{\partial \tau^{\alpha}}+\frac{\partial^{\alpha} \Upsilon_{0}(\xi, \tau)}{\partial \tau^{\alpha}}+C_{1} \frac{\partial^{\alpha} \Upsilon_{1}(\xi, \tau)}{\partial \tau^{\alpha}} \\
& +C_{2} \Upsilon_{0}(\xi, \tau) \frac{\partial \Upsilon_{0}(\xi, \tau)}{\partial x} \xi+C_{1} \Upsilon_{0}(\xi, \tau) \frac{\partial \Upsilon_{0}(\xi, \tau)}{\partial \xi} \\
& +C_{1} \Upsilon_{0}(\xi, \tau) \frac{\partial \Upsilon_{1}(\xi, \tau)}{\partial \xi}+C_{2} \frac{\partial^{3} \Upsilon_{0}(\xi, \tau)}{\partial \xi^{3}} \\
& +C_{1} \frac{\partial^{3} \Upsilon_{1}(\xi, \tau)}{\partial \xi^{3}}-C_{2} \frac{\partial^{5} \Upsilon_{1}(\xi, \tau)}{\partial \xi^{5}}
\end{aligned}
$$

Apply the inverse operator $I^{\alpha}$, the solution of the above problems is given as follows:

$$
\begin{gathered}
\Upsilon_{0}(\xi, \tau)=\frac{105}{169} \operatorname{sech}^{4}\left(\frac{\xi}{2 \sqrt{13}}\right), \\
\Upsilon_{1}\left(\xi, \tau, C_{1}\right)=\frac{-7560 C_{1} \tau^{\alpha} \operatorname{sech}^{4}(\xi / 2 \sqrt{13}) \tan (\xi / 2 \sqrt{13})}{28561 \sqrt{13} \Gamma(1+\alpha)}, \\
\Upsilon_{2}\left(\xi, \tau, C_{1}, C_{2}\right)=\frac{1}{62748517 \Gamma(1+\alpha)} \\
\cdot\left(1890 e^{-\xi / \sqrt{13}} \tau^{\alpha}\left(-169 \sqrt{13}\left(C_{1}+C_{1}^{2}+C_{2}\right)\left(-1+e^{2 \xi / \sqrt{13}}\right)\right)\right. \\
\left.\left.+\frac{92^{3-2 \alpha C_{1}^{2}}\left(1-3 e^{\xi / \sqrt{13}}+e^{2 \xi / \sqrt{13}}\right) \sqrt{\pi} \tau^{\alpha}}{\sqrt{13} \Gamma(1+\alpha)}\right) \operatorname{sech}^{6}\left(\frac{\xi}{2 \sqrt{13}}\right)\right) .
\end{gathered}
$$

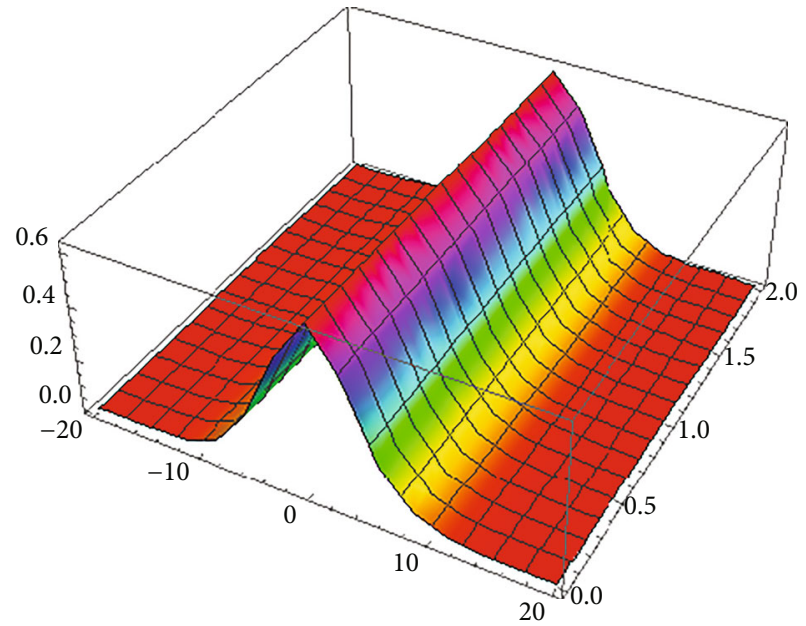

FIgURE 1: 3D surface obtained by OHAM solution for fractional Kawahara equation at $\alpha=0.5$.

The second-order OHAM solution is presented as follows:

$$
\tilde{\Upsilon}\left(\xi, \tau, C_{i}\right)=\Upsilon_{0}(\xi, \tau)+\Upsilon_{1}\left(\xi, \tau, C_{1}\right)+\Upsilon_{2}\left(\xi, \tau, C_{1}, C_{2}\right) .
$$

For $\alpha=1$, second-order OHAM solution for Kawahara equation is 


$$
\tilde{Y}(\xi, \tau)=\frac{105 \operatorname{sech}^{4}(\xi / 2 \sqrt{13})\left(371293+72 \tau\left(-9 C_{1}^{2} \tau\left(-4+5 \operatorname{sech}^{2}(\xi / 2 \sqrt{13})-169 \sqrt{13}\left(C_{1}\left(2+C_{1}\right)+C_{2}\right) \tanh (\xi / 2 \sqrt{13})\right)\right.\right.}{62748517} .
$$

For $\alpha=2 / 3$, second-order OHAM solution for Kawahara equation is

$$
\tilde{Y}(\xi, \tau)=\frac{105 \operatorname{sech}^{4}(\xi / 2 \sqrt{13})\left(371293+72 \tau^{2 / 3}\left(-18 C_{1}^{2} \tau^{2 / 3}\left(-4+5 \operatorname{sech}^{2}(\xi / 2 \sqrt{13})\right) / \Gamma(7 / 3)-169 \sqrt{13}\left(C_{1}\left(2+C_{2}\right) \tanh (\xi / 2 \sqrt{13}) / \Gamma(5 / 3)\right)\right)\right.}{62748517} .
$$

4.2. Numerical Solution of Fractional Modified Kawahara Equation. Assume the following time-fractional modified Kawahara system presented by

$$
\frac{\partial^{\alpha} \Upsilon(\xi, \tau)}{\partial \tau^{\alpha}}+\Upsilon^{2}(\xi, \tau) \frac{\partial \Upsilon(\xi, \tau)}{\partial \xi}+p \frac{\partial^{3} \Upsilon(\xi, \tau)}{\partial \xi^{3}}+q \frac{\partial^{5} \Upsilon(\xi, \tau)}{\partial \xi^{5}}=0,0<\alpha \leq 1,
$$

with I.C.,

$$
\Upsilon(\xi, 0)=\frac{3 \rho}{\sqrt{-10 \mu}} \operatorname{sech}^{2}(k \xi), k=\frac{1}{2} \sqrt{\frac{-\rho}{2 \mu}} .
$$

When $\alpha=1$, the exact solution is given by [35] as

$$
\Upsilon(\xi, \tau)=\frac{3 \rho}{\sqrt{-10 \mu}} \operatorname{sech}^{2}\left(k(\xi-\psi t), \psi=\frac{25 \mu-4 \rho^{2}}{25 \mu} .\right.
$$

Following the OHAM procedure, we have the following.

$$
\begin{aligned}
\Upsilon_{0}(\xi, \tau) & =\frac{3 \rho}{\sqrt{-10 \mu}} \operatorname{sech}^{2}\left(\left(\frac{1}{2} \sqrt{\frac{-\rho}{2 \mu}}\right) \xi\right), \\
\Upsilon_{1}\left(\xi, \tau, C_{1}\right) & =\frac{3 C_{1} \rho 7 / 2 \tau^{\alpha} \operatorname{sech}^{5}(\sqrt{\rho} \xi / 2 \sqrt{5} \sqrt{\mu})(-59 \sin (\sqrt{\rho} \xi / 2 \sqrt{5} \sqrt{\mu})+\sin (\sqrt{\rho} \xi / 2 \sqrt{5} \sqrt{\mu})) \tan (\xi / 2 \sqrt{13})}{\left.500 \sqrt{-\mu} \mu^{3 / 2} \sqrt{2} \alpha \Gamma \alpha\right)} .
\end{aligned}
$$

Zero-order problem:

$$
\frac{\partial^{\alpha} \Upsilon_{0}(\xi, \tau)}{\partial \tau^{\alpha}}=0, \Upsilon_{0}(\xi, 0)=\frac{3 \rho}{\sqrt{-10 \mu}} \operatorname{sech}^{2}(k \xi), k=\frac{1}{2} \sqrt{\frac{-\rho}{2 \mu}} .
$$

First-order problem:

$$
\begin{aligned}
\frac{\partial^{\alpha} \Upsilon_{1}(\xi, \tau)}{\partial \tau^{\alpha}}= & \frac{\partial^{\alpha} \Upsilon_{0}(\xi, \tau)}{\partial \tau^{\alpha}}+C_{1} \frac{\partial^{\alpha} \Upsilon_{0}(\xi, \tau)}{\partial \tau^{\alpha}}-C_{1} \Upsilon_{0} \frac{\partial \Upsilon_{0}(\xi, \tau)}{\partial \xi} \\
& +C_{1} \frac{\partial^{3} \Upsilon_{0}(\xi, \tau)}{\partial \xi^{3}}-C_{1} \frac{\partial^{5} \Upsilon_{0}(\xi, \tau)}{\partial \xi^{5}} .
\end{aligned}
$$

Apply the inverse operator $I^{\alpha}$, the solution of the above problems is given as follows:
The $1^{\text {st }}$-order OHAM solution is given by the following expression:

$$
\tilde{\Upsilon}\left(\xi, \tau, C_{i}\right)=\Upsilon_{0}(\xi, \tau)+\Upsilon_{1}\left(\xi, \tau, C_{1}\right) .
$$

\section{Results and Discussion}

We implemented OHAM to provide approximate numerical solutions to fractional and modified Kawahara equations.
Numerical values are tabulated for the auxiliary constants in Tables 1 and 2 for Kawahara and modified Kawahara equations at various values of $\alpha$. Table 3 gives the estimation of the second-order OHAM solution and the third-order NIM solution for the Kawahara fractional equation. Table 4 compares the absolute errors of the second-order OHAM solution for various $\alpha$ values. Table 4 presents the values of the first-order OHAM solution and the thirdorder NIM solution for the various values of $\alpha$. Table 5 


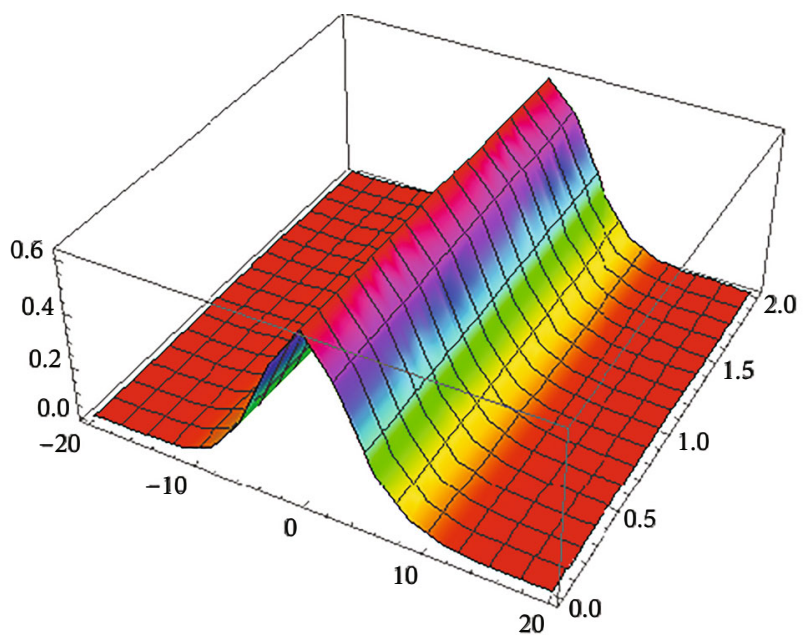

FIGURE 2: 3D surface obtained by OHAM solution for fractional Kawahara equation at $\alpha=1$.

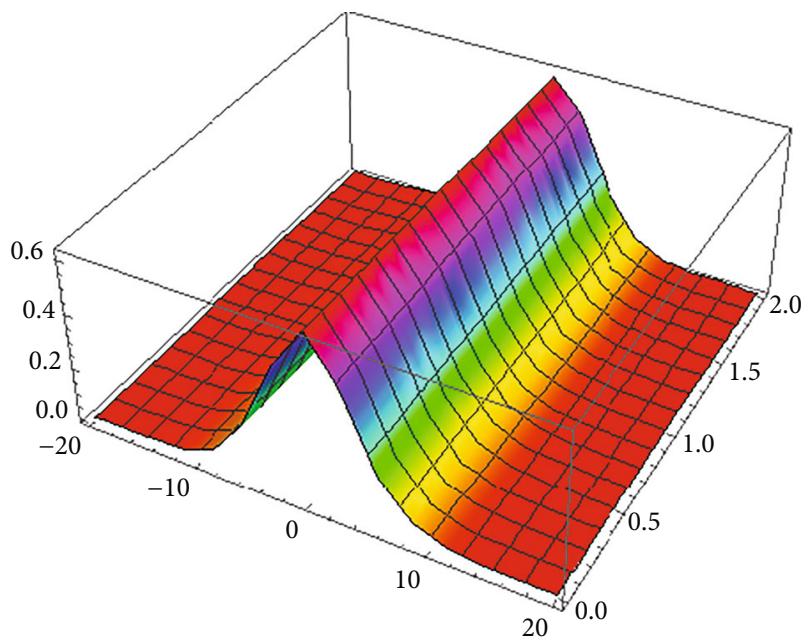

FIGURE 3: 3D surface obtained by exact solution for fractional Kawahara equation at $\alpha=1$.

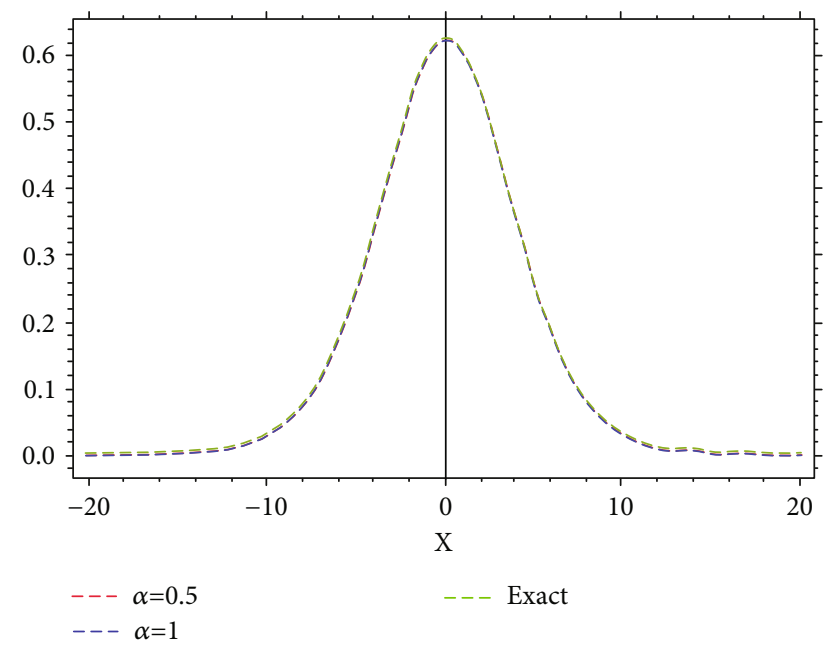

FIGURE 4: The curves show the comparison between exact solution and approximate solution for different values of $\alpha$ at $\tau=0.5$ for Kawahara equation.

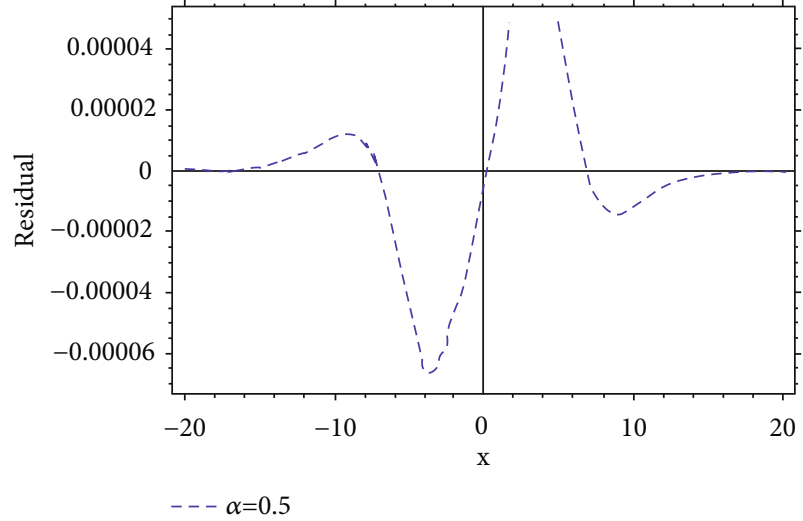

FIgURE 5: Residual obtained by OHAM for $\alpha=0.5$ at $\tau=0.5$, for Kawahara equation.

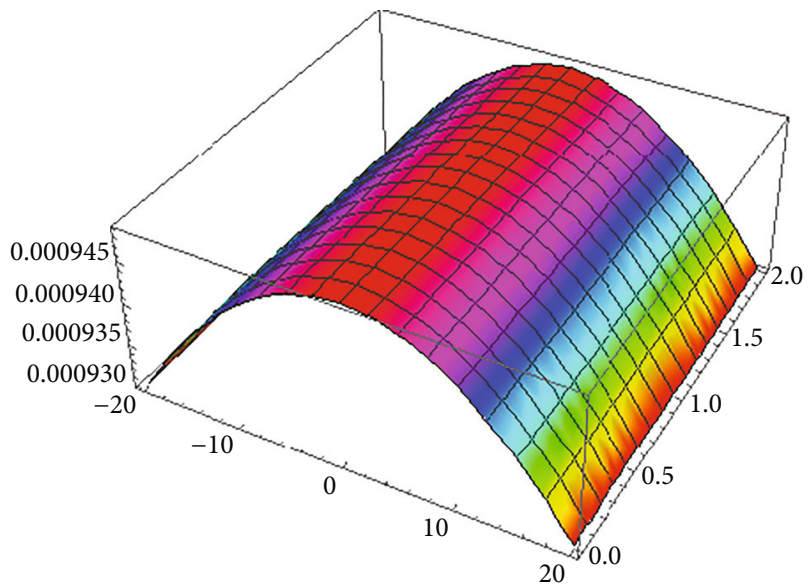

Figure 6: 3D plot of OHAM solution for fractional modified Kawahara equation at $\alpha=0.5$.

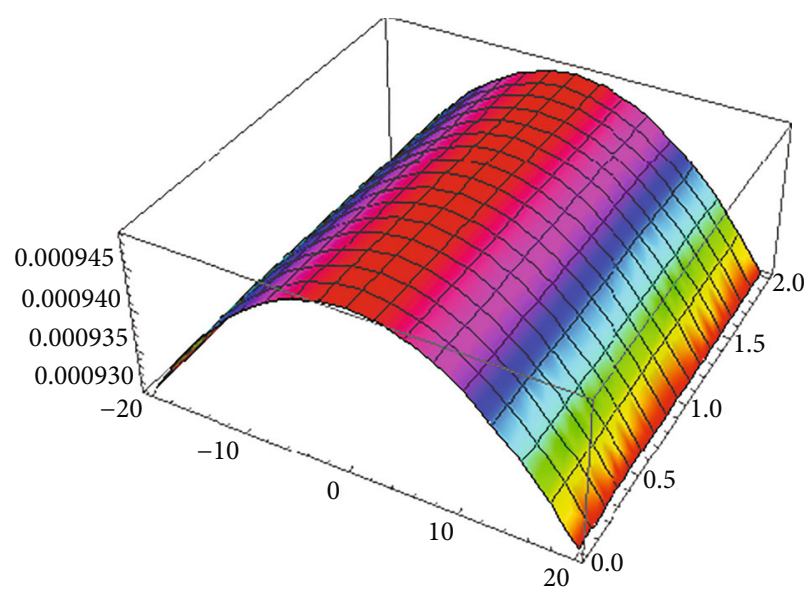

FIgURE 7: 3D surface obtained by OHAM solution for fractional modified Kawahara equation at $\alpha=1$.

compares first-order OHAM solution with third-order NIM solution for time-fractional modified Kawahara equations for different values of $\alpha$.

Figures 1-3 depict the 3D surfaces obtained by secondorder OHAM as well as the accurate solutions to fractional 


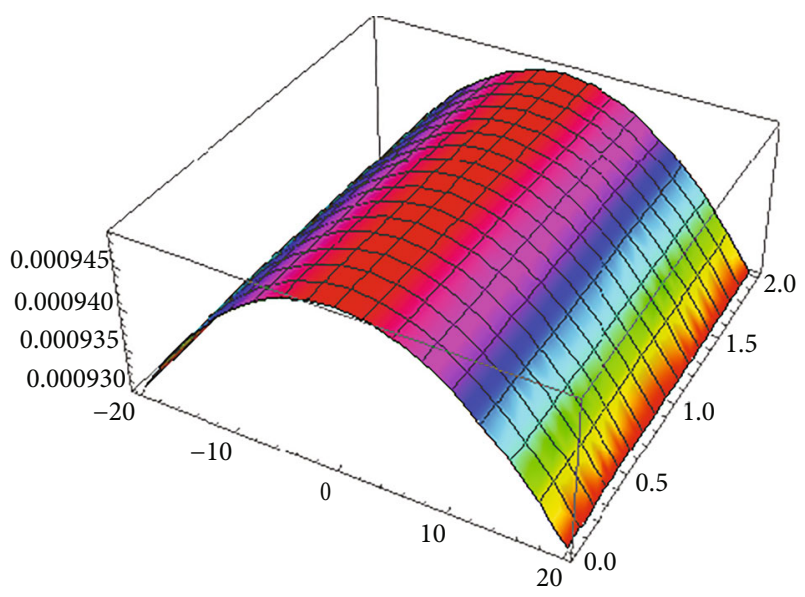

FIGURE 8: 3D surface obtained by exact solution for fractional modified Kawahara equation at $\alpha=1$.

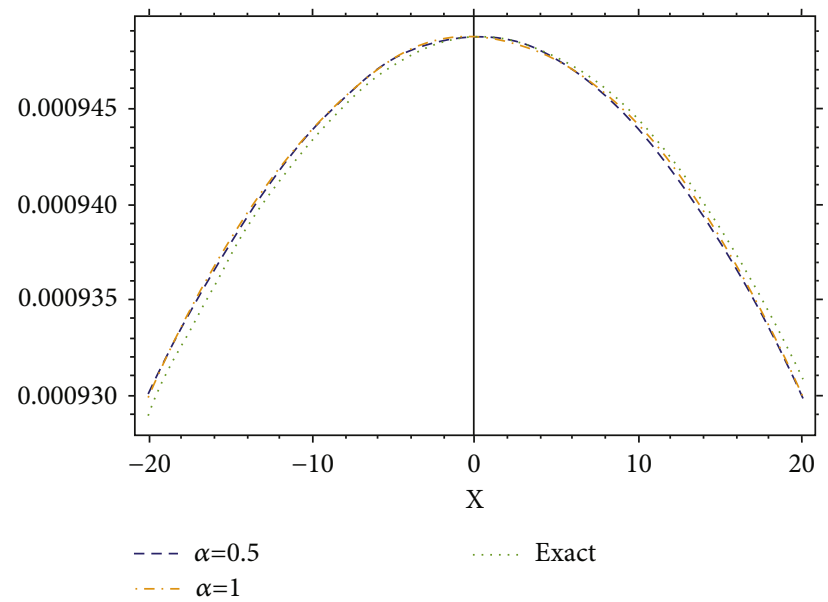

Figure 9: The curves show the comparison between exact solution and approximate solution for different values of $\alpha$ at $\tau=0.5$ for modified Kawahara equation.

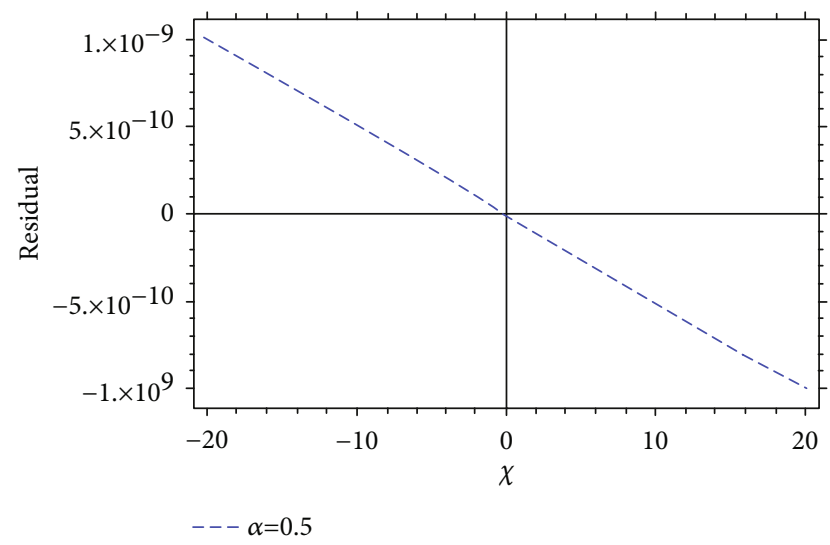

FIgURE 10: Residual obtained by OHAM for $\alpha=0.5$ at $\tau=0.5$, for modified Kawahara equation.
Kawahara equation at $\alpha=0.5$ and 1 . Figure 4 shows the residual for $\alpha=0.5$, whereas Figure 5 shows the 2D surface of the second-order OHAM solution for various values of $\alpha$. Figures 6-8 show the 3D plots for the first-order OHAM solution and exact solution for the fractional modified Kawahara equation at $\alpha=0.5$ and 1 . Figure 9 depicts a two-dimensional graph of the first-order OHAM solution for different values of $\alpha$. The residual for $\alpha=0.5$ is shown in Figure 10.

The results obtained by the second-order OHAM solution for the Kawahara fractional equation agree with both the closed and the NIM solution. Similarly, for fractional modified Kawahara equation, the results achieved by the first-order OHAM solutions are exactly the same as for the third-order NIM solutions.

\section{Conclusions}

We observe that OHAM converges rapidly towards the closed solution with a lower sequence of approximation of fractional orders of the Kawahara equations and modified Kawahara equations based on the calculated results. The results achieved with the proposed approach are highly encouraging compared to the new iterative method (NIM). This proposed approach is capable of providing the greatest accuracy within the lowest approximation sequence. This approach does not require choices between small and large parameter assumptions in problems. The results are analyzed and explained with the help of graphs by considering different values of parameters. Results reveal that as the value of fractional-order derivatives approaches to 1 , the approximate solution converges to the exact solution. The convergence of this approach is independent of initial assumptions. The precision of the proposed approach can be improved by assuming high approximations, and therefore, it may be highly attractive for researchers to use our approach to solve fractional-order systems emerging in the science of technology.

\section{Data Availability}

There is no data for this study.

\section{Conflicts of Interest}

The authors have declared no conflict of interest.

\section{Acknowledgments}

This research was funded by Princess Nourah bint Abdulrahman University Researchers Supporting Project number (PNURSP2022R229), Princess Nourah bint Abdulrahman University, Riyadh, Saudi Arabia.

\section{References}

[1] I. Podlubny, Fractional Differential Equations: An Introduction to Fractional Derivatives, Fractional Differential Equations, to Methods of Their Solution and Some of Their Applications, Elsevier, 1998. 
[2] L. Debnath, "Recent applications of fractional calculus to science and engineering," International Journal of Mathematics and Mathematical Sciences, vol. 2003, no. 54, pp. 3413-3442, 2003.

[3] K. S. Miller and B. Ross, An Introduction to the Fractional Calculus and Fractional Differential Equations, Wiley, 1993.

[4] F. Calculus, Integrations and Differentiations of Arbitrary Order, K. Nishimoto, 1989.

[5] A. Ali, S. Islam, M. R. Khan et al., "Dynamics of a fractional order Zika virus model with mutant," Alexandria Engineering Journal, vol. 61, no. 6, pp. 4821-4836, 2022.

[6] X.-H. Zhang, A. Ali, M. A. Khan, M. Y. Alshahrani, T. Muhammad, and S. Islam, "Mathematical analysis of the TB model with treatment via Caputo-type fractional derivative," Discrete Dynamics in Nature and Society, vol. 2021, 15 pages, 2021.

[7] A. Ali, F. S. Alshammari, S. Islam, M. A. Khan, and S. Ullah, "Modeling and analysis of the dynamics of novel coronavirus (COVID-19) with Caputo fractional derivative," Results in Physics, vol. 20, article 103669, 2021.

[8] M. A. Aba Oud, A. Ali, H. Alrabaiah, S. Ullah, M. A. Khan, and S. Islam, "A fractional order mathematical model for COVID19 dynamics with quarantine, isolation, and environmental viral load," Advances in Difference Equations, vol. 2021, no. 1, pp. 106-119, 2021.

[9] A. Rehman, Z. Salleh, and T. Gul, "Soot formation characteristics of homogeneous supercritical fuel spray of dieseline blend," Journal of Advanced Research in Fluid Mechanics and Thermal Sciences, vol. 81, no. 2, pp. 67-81, 2021.

[10] G. Adomian, Solving Frontier Problems of Physics: The Decomposition Method, vol. 60, Springer Science \& Business Media, 2013.

[11] G. Adomian and R. Rach, "Modified Adomian polynomials," Mathematical and Computer Modelling, vol. 24, no. 11, pp. 39-46, 1996.

[12] J. H. He, "Variational iteration method - a kind of non-linear analytical technique: some examples," International Journal of Non-Linear Mechanics, vol. 34, no. 4, pp. 699-708, 1999.

[13] N. H. Sweilam and M. M. Khader, "Variational iteration method for one dimensional nonlinear thermoelasticity," Chaos, Solitons \& Fractals, vol. 32, no. 1, pp. 145-149, 2007.

[14] J. H. He, "Homotopy perturbation technique," Computer Methods in Applied Mechanics and Engineering, vol. 178, no. 3-4, pp. 257-262, 1999.

[15] S. Abbasbandy, "The application of homotopy analysis method to nonlinear equations arising in heat transfer," Physics Letters A, vol. 360, no. 1, pp. 109-113, 2006.

[16] W. Deng, "Finite Element Method for the Space and Time Fractional Fokker-Planck Equation," SIAM Journal on Numerical Analysis, vol. 47, no. 1, pp. 204-226, 2009.

[17] V. Daftardar-Gejji and H. Jafari, "An iterative method for solving nonlinear functional equations," Journal of Mathematical Analysis and Applications, vol. 316, no. 2, pp. 753-763, 2006.

[18] V. Daftardar-Gejji and S. Bhalekar, "Solving multi-term linear and non-linear diffusion-wave equations of fractional order by Adomian decomposition method," Fractional Calculus and Applied Analysis, vol. 202, no. 1, pp. 113-120, 2008.

[19] A. Saeed, P. Kumam, T. Gul, W. Alghamdi, W. Kumam, and A. Khan, "Darcy-Forchheimer couple stress hybrid nanofluids flow with variable fluid properties," Scientific Reports, vol. 11, no. 1, pp. 1-13, 2021.
[20] Y. Cao, H. Ayed, T. Abdulrazzaq, T. Gul, A. Bariq, and B. Bouallegue, "Effect of the number of nozzles of swirl flow generator utilized in flat plate solar collector: an entropic analysis," International Journal of Photoenergy, vol. 2021, 10 pages, 2021.

[21] A. Saeed, P. Kumam, S. Nasir, T. Gul, and W. Kumam, "Nonlinear convective flow of the thin film nanofluid over an inclined stretching surface," Scientific Reports, vol. 11, no. 1, pp. 1-15, 2021.

[22] V. Marinca, N. Herişanu, and I. Nemeş, "Optimal homotopy asymptotic method with application to thin film flow," Open Physics, vol. 6, no. 3, pp. 648-653, 2008.

[23] V. Marinca and N. Herişanu, "The optimal homotopy asymptotic method for solving Blasius equation," Applied Mathematics and Computation, vol. 231, pp. 134-139, 2014.

[24] N. Herisanu, V. Marinca, and G. Madescu, “An analytical approach to non-linear dynamical model of a permanent magnet synchronous generator," Wind Energy, vol. 18, no. 9, pp. 1657-1670, 2015.

[25] V. Marinca and N. Herişanu, "Application of optimal homotopy asymptotic method for solving nonlinear equations arising in heat transfer," International Communications in Heat and Mass Transfer, vol. 35, no. 6, pp. 710-715, 2008.

[26] V. Marinca, N. Herișanu, C. Bota, and B. Marinca, “An optimal homotopy asymptotic method applied to the steady flow of a fourth- grade fluid past a porous plate," Applied Mathematics Letters, vol. 22, no. 2, pp. 245-251, 2009.

[27] S. Sarwar, S. Alkhalaf, S. Iqbal, and M. A. Zahid, "A note on optimal homotopy asymptotic method for the solutions of fractional order heat- and wave-like partial differential equations," Computers \& Mathematics with Applications, vol. 70, no. 5, pp. 942-953, 2015.

[28] S. Sarwar and M. M. Rashidi, "Approximate solution of twoterm fractional-order diffusion, wave-diffusion, and telegraph models arising in mathematical physics using optimal homotopy asymptotic method," Waves in Random and Complex Media, vol. 26, no. 3, pp. 365-382, 2016.

[29] R. Nawaz, L. Zada, A. Khattak, M. Jibran, and A. Khan, “Optimum solutions of fractional order Zakharov-Kuznetsov equations," Complexity, vol. 2019, 9 pages, 2019.

[30] L. Zada, R. Nawaz, and S. S. Bushnaq, "An efficient approach for solution of fractional order differential-difference equations arising in nanotechnology," Applied Mathematics ENotes, vol. 20, pp. 297-307, 2020.

[31] L. Zada and R. Nawaz, "Solution of time-fractional order RLW equation using optimal homotpy asymptotic method," AIP Conference Proceedings, vol. 2116, no. 1, p. 300005, 2019.

[32] N. A. Kudryashov, "A note on new exact solutions for the Kawahara equation using Exp-function method," Journal of Computational and Applied Mathematics, vol. 234, no. 12, pp. 3511-3512, 2010.

[33] D. Kaya and K. Al-Khaled, "A numerical comparison of a Kawahara equation," Physics Letters A, vol. 363, no. 5-6, pp. 433-439, 2007.

[34] J. Lu, "Analytical approach to Kawahara equation using variational iteration method and homotopy perturbation method," Topological Methods in Nonlinear Analysis, vol. 31, no. 2, pp. 287-293, 2008.

[35] B. R. Sontakke and A. Shaikh, "Approximate solutions of time fractional Kawahara and modified Kawahara equations by fractional complex transform," Communications in numerical analysis, vol. 2016, no. 2, pp. 218-229, 2016. 